\title{
Front Matter: Volume 7140
}

, "Front Matter: Volume 7140," Proc. SPIE 7140, Lithography Asia 2008, 714001 (12 December 2008); doi: 10.1117/12.820873

SPIE. Event: SPIE Lithography Asia - Taiwan, 2008, Taipei, Taiwan 


\title{
PROCEEDINGS OF SPIE
}

\section{Lithography Asia 2008}

\author{
Alek C. Chen \\ Burn Lin \\ Anthony Yen \\ Editors
}

4-6 November 2008

Taipei, Taiwan

Sponsored and Published by

SPIE

Cooperating Organization

TSIA-Taiwan Semiconductor Industry Association 
The papers included in this volume were part of the technical conference cited on the cover and title page. Papers were selected and subject to review by the editors and conference program committee. Some conference presentations may not be available for publication. The papers published in these proceedings reflect the work and thoughts of the authors and are published herein as submitted. The publisher is not responsible for the validity of the information or for any outcomes resulting from reliance thereon.

Please use the following format to cite material from this book:

Author(s), "Title of Paper," in Lithography Asia 2008, edited by Alek C. Chen, Burn Lin, Anthony Yen, Proceedings of SPIE Vol. 7140 (SPIE, Bellingham, WA, 2008) Article CID Number.

ISSN 0277-786X

ISBN 9780819473813

Published by

SPIE

P.O. Box 10, Bellingham, Washington 98227-0010 USA

Telephone +1 3606763290 (Pacific Time) · Fax +1 3606471445

SPIE.org

Copyright () 2008, Society of Photo-Optical Instrumentation Engineers

Copying of material in this book for internal or personal use, or for the internal or personal use of specific clients, beyond the fair use provisions granted by the U.S. Copyright Law is authorized by SPIE subject to payment of copying fees. The Transactional Reporting Service base fee for this volume is $\$ 18.00$ per article (or portion thereof), which should be paid directly to the Copyright Clearance Center (CCC), 222 Rosewood Drive, Danvers, MA 01923. Payment may also be made electronically through CCC Online at copyright.com. Other copying for republication, resale, advertising or promotion, or any form of systematic or multiple reproduction of any material in this book is prohibited except with permission in writing from the publisher. The CCC fee code is 0277-786X/08/\$18.00.

Printed in the United States of America.

Publication of record for individual papers is online in the SPIE Digital Library.

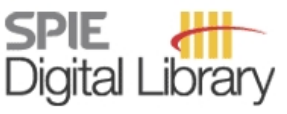

SPIEDigitalLibrary.org

Paper Numbering: Proceedings of SPIE follow an e-First publication model, with papers published first online and then in print and on CD-ROM. Papers are published as they are submitted and meet publication criteria. A unique, consistent, permanent citation identifier (CID) number is assigned to each article at the time of the first publication. Utilization of CIDs allows articles to be fully citable as soon they are published online, and connects the same identifier to all online, print, and electronic versions of the publication. SPIE uses a six-digit CID article numbering system in which:

- The first four digits correspond to the SPIE volume number.

- The last two digits indicate publication order within the volume using a Base 36 numbering system employing both numerals and letters. These two-number sets start with 00, 01, 02, 03, 04 , $05,06,07,08,09,0 A, 0 B \ldots$. OZ, followed by 10-1Z, 20-2Z, etc.

The CID number appears on each page of the manuscript. The complete citation is used on the first page, and an abbreviated version on subsequent pages. Numbers in the index correspond to the last two digits of the six-digit CID number. 


\section{Contents}

\section{EUVL TECHNOLOGY}

714006 EUVL contamination control studies for high-volume manufacturing (Invited Paper) [7140-05] D. N. Ruzic, R. Raju, J. Sporre, H. Shin, W. M. Lytle, M. J. Neumann, Univ. of Illinois at Urbana-Champaign (United States)

714007 Full field EUV lithography: lessons learned on EUV ADT imaging, EUV resist, and EUV reticles [7140-06]

E. Hendrickx, A. M. Goethals, IMEC (Belgium); A. Niroomand, IMEC (Belgium) and Micron Technology, Inc. (Belgium); R. Jonckheere, F. Van Roey, G. F. Lorusso, J. Hermans, B. Baudemprez, K. Ronse, IMEC (Belgium)

714008 Current benchmarking results of EUV resist at Selete [7140-07]

D. Kawamura, K. Kaneyama, S. Kobayashi, H. Oizumi, T. Itani, Semiconductor Leading Edge Technologies, Inc. (Japan)

714009 Methodology of flare modeling and compensation in EUVL [7140-08]

I. Kim, SAMSUNG Electronics Co., Ltd. (Korea, Republic of); H. Kang, ASML (Korea, Republic of); C. Park, J.-O. Park, J. Lee, J. Park, D. Goo, J. Yeo, S.-W. Choi, W. Han, SAMSUNG Electronics Co., Ltd. (Korea, Republic of)

\section{EMERGENT LITHOGRAPHIC TECHNOLOGY}

7140 OA What is the strongest candidate in lithography for $2 x \mathbf{~ n m}$ HP and beyond? (Invited Paper) [7140-09]

K. Hashimoto, I. Yoneda, T. Koshiba, S. Mikami, T. Ota, M. Ito, T. Nakasugi, T. Higashiki, Toshiba Corp. (Japan)

7140 OC Nano-imprint fabrication and light extraction simulation of photonic crystals on OLED [7140-11]

J. W.-C. Yu, National Cheng Kung Univ. (Taiwan); Y.-B. Guo, Toppan CFI Co., Ltd. (Taiwan); J.-Y. Chen, Hsing Kuo Univ. of Management (Taiwan); F. C.-N. Hong, National Cheng Kung Univ. (Taiwan)

\section{METROLOGY}

7140 OF The CD metrology perspectives and future trends (Invited Paper) [7140-14] J. Foucher, CEA-LETI (France); E. Pargon, M. Martin, LTM/CNRS (France); V. Farys, STMicroelectronics (France); S. Bécu, CEA-LETI (France); L. Babaud, LTM/CNRS (France) and STMicroelectronics (France) 
$71400 \mathrm{O}$ Optimizing integrated optical CD monitoring by floating pre-process variations in a complex multi-layer structure [7140-15]

M. Strobl, L. Huang, Inotera Memories Inc. (Taiwan); A. Li, Tokyo Electron Taiwan Ltd. (Taiwan); Y. Luo, Y. Wen, Timbre Technology, Inc. (United States)

$7140 \mathrm{OH} \quad$ Improving sidewall profile metrology with enhanced 3D-AFM [7140-16]

T. Bao, L. Mininni, D. Dawson, Veeco Instruments Inc. (United States)

7140 Ol Accurate dimensional characterization of periodic structures by spectroscopic Mueller polarimetry [7140-17]

M. Foldyna, A. De Martino, Lab. de Physique des Interfaces et des Caurel Minces, CNRS,

Ecole Polytechnique (France); D. Cattelan, Groupe HORIBA (France); F. Bogeat, C. Licitra, J. Foucher, P. Barritault, J. Hazart, CEA-LETI, MINATEC (France)

7140 0J Characterization of inhomogeneous samples by spectroscopic Mueller polarimetry [7140-18]

M. Foldyna, A. De Martino, R. Ossikovski, E. Garcia-Cavrel, CNRS, Lab. de Physique des Interfaces et des Caurel Minces, Ecole Polytechnique (France); D. Cattelan, Groupe HORIBA (France); C. Licitra, CEA LETI MINATEC (France)

\section{PROCESS CONTROL AND METROLOGY}

7140 OK An update on the DPL overlay discontinuity (Invited Paper) [7140-19] M. Adel, KLA-Tencor Corp., Israel (Israel)

7140 OM Advance overlay correction beyond 32nm DRAM process [7140-21] C. T. Hung, C. P. Hsia, T. S. Cheng, C. Y. Huang, W. B. Wu, C. L. Shih, Nanya Technologies Corp. (Taiwan)

7140 ON A comprehensive look at a new metrology technique to support the needs of lithography performance in near future [7140-22]

J. Hu, C.-M. Ke, W. Wang, J. Huang, H. L. Chung, Taiwan Semiconductor Manufacturing Co. Ltd. (Taiwan); C. R. Liang, V. Shih, H. H. Liu, H. J. Lee, L. G. Terng, Y. D. Fan, Taiwan Semiconductor Manufacturing Co. Ltd. (Taiwan); M. van der Schaar, K. Yuan, V. Wang, C. Wang, M. Shahrjerdy, A. Fuchs, K. Bhattacharyya, K. van der Mast, ASML (Netherlands)

LITHOGRAPHY PROCESS CONTROL

$71400 Q \quad$ Focus and dose control for high volume manufacturing (Invited Paper) [7140-25] H. Ina, K. Sentoku, Canon Inc. (Japan)

7140 OR Lithography hotspot discovery at 70nm DRAM 300mm fab: process window qualification using design base binning [7140-26]

D. Chen, Inotera Memories Inc. (Taiwan); D. Chen, KLA-Tencor Corp. (United States); R. Yen, M. Cheng, A. Lan, Inotera Memories Inc. (Taiwan); R. Ghaskadvi, KLA-Tencor Corp. (United States) 
7140 OS Accelerating 32nm BEOL technology development by advanced wafer inspection methodology [7140-27]

P. R. Jeng, C. L. Lin, S. Jang, M. S. Liang, Taiwan Semiconductor Manufacturing Co., Ltd. (Taiwan); W. Chen, D. Tsui, D. Chen, H. Chen, C. Young, E. Chang, KLA-Tencor Corp. (United States)

$7140 \mathrm{OU}$ Reducing capital and labor costs of 193nm lithography monitoring of airborne molecular contamination (AMC) through proactive assessment and implementation of AMC monitoring techniques and strategies [7140-29]

S. Rowley, Particle Measuring Systems, Inc. (United States); J. Yang, A. Wei, Sunway Scientific Corp. (Taiwan)

\section{DEFECT INSPECTION}

7140 OW Advanced inspection methodologies for detection and classification of killer substrate defects [7140-31]

A. Chen, V. Huang, S. Chen, C. J. Tsai, K. Wu, Taiwan Semiconductor Manufacturing Co. Ltd. (Taiwan); H. Zhang, KLA-Tencor Corp. (United States); K. Sun, KLA-Tencor Taiwan (Taiwan); J. Saito, KLA-Tencor Corp. (United States); H. Chen, D. Hu, KLA-Tencor Taiwan (Taiwan); M. Li, W. Shen, U. Mahajan, KLA-Tencor Corp. (United States)

7140 0X Electron beam charging of a $\mathrm{SiO}_{2}$ layer on Si: a comparison between Monte Carlo-simulated and experimental results [7140-32]

K. Inai, K. Ohya, H. Kuwada, R. Kawasaki, Univ. of Tokushima (Japan); M. Saito, K. Fujihara, T. Hayashi, Tokyo Electron Ltd. (Japan); J. Jau, K. Kanai, Hermes Microvision, Inc. (United States)

7140 OY Advanced technology for after-develop inspection [7140-33]

Z. Y. Chen, I. C. Chou, J. H. Yang, Taiwan Semiconductor Manufacturing Co., Ltd. (Taiwan); W. Chen, J. Chang, H. Chen, M. Ng, M.-C. Wu, C. Perry-Sullivan, M. Li, KLA-Tencor Corp. (Taiwan)

OPTICAL (IMAGING)

714010 An innovative Source-Mask co-Optimization (SMO) method for extending low $k_{1}$ imaging [7140-35]

S. Hsu, L. Chen, Z. Li, S. Park, K. Gronlund, H. Liu, N. Callan, ASML Brion Technologies, Inc. (United States); R. Socha, S. Hansen, ASML TDC (United States)

714011 A novel lithography design and verification methodology with patterning failure [7140-36] S. Miyoshi, Y. Kobayashi, S. Tanaka, K. Kawano, K. Hashimoto, S. Inove, Toshiba Corp. (Japan)

714012 A new calibration method for latent image fidelity [7140-37]

E. Barouch, S. L. Knodle, Boston Univ. (United States)

714013 An imaging system for extended ArF immersion lithography [7140-38]

T. Matsuyama, Y. Ohmura, T. Nakashima, Y. Uehara, Nikon Corp. (Japan) 
714014 Model-based sub-resolution assist features using an inverse lithography method (Best Student Paper Award) [7140-83]

J.-C. YU, P. YU, H.-Y. Chao, National Chiao Tung Univ. (Taiwan)

\section{OPTICAL (IMAGING) II}

714015 Immersion lithography: its history, current status, and future prospects [7140-40]

S. Owa, H. Nagasaka, Nikon Corp. (Japan)

714017 Patterning performance of hyper NA immersion lithography for $32 \mathrm{~nm}$ node logic process [7140-42]

K. Takahata, M. Kajiwara, Y. Kitamura, T. Ojima, M. Satake, H. Fujise, Y. Seino, T. Ema, M. Takakuwa, S. Nakagawa, T. Kono, M. Asano, S. Kyo, A. Nomachi, H. Harakawa, T. Ishida, S. Hasegawa, K. Miyashita, Toshiba Corp. (Japan); T. Murakami, S. Nagahara, K. Takeda, NEC Electronics Corp. (Japan); S. Mimotogi, S. Inove, Toshiba Corp. (Japan)

714018 Orientation Zernike Polynomials: a systematic description of polarized imaging using high NA lithography lenses [7140-43]

T. Heil, J. Ruoff, J. T. Neumann, M. Totzeck, D. Krähmer, Carl Zeiss SMT AG (Germany); B. Geh, Carl Zeiss SMT AG (Germany) and ASML US, Inc. (United States); P. Gräupner, Carl Zeiss SMT AG (Germany)

714019 Resist-based polarization monitoring for 193nm high-numerical aperture lithography [7140-44]

R. Tu, Benchmark Technologies (United States); G. McIntyre, IBM Advanced Lithography Research (United States)

7140 1A Binary mask optimization for inverse lithography with partially coherent illumination [7140-87]

X. Ma, G. R. Arce, Univ. of Delaware (United States)

7140 1B Extending KrF lithography beyond 80nm with the TWINSCAN XT:1000H 0.93NA scanner [7140-46]

W. de Boeij, G. Dicker, M. de Wit, F. Bornebroek, M. Zellenrath, H.-J. Voorma, B. Smeets,

R. Toussaint, B. Paarhuis, M. de Jong, ASML Netherlands B.V. (Netherlands); D. Hellweg,

K. Kornitzer, Carl Zeiss SMT AG (Germany)

\section{EUV SCANNER AND SOURCES}

7140 1C Development of EUV lithography tools at Nikon (Invited Paper) [7140-47]

K. Murakami, T. Oshino, H. Kondo, H. Chiba, K. Nomura, H. Kawai, Y. Kohama, K. Morita, K. Hada, Y. Ohkubo, T. Miura, Nikon Corp. (Japan)

$71401 \mathrm{E} \quad$ Laser-produced plasma source system development [7140-49]

D. C. Brandt, I. V. Fomenkov, A. I. Ershov, W. N. Partlo, D. W. Myers, N. R. Böwering,

G. O. Vaschenko, O. V. Khodykin, A. N. Bykanov, J. R. Hoffman, C. P. Chrobak,

S. N. Srivastava, D. A. Vidusek, S. De Dea, R. R. Hou, Cymer, Inc. (United States) 
$7140 \mathrm{IF}$ Status of DPP EUV sources development for Beta/HVM [7140-50]

M. Yoshioka, XTREME technologies GmbH (Germany); P. Zink, Philips Extreme UV GmbH (Germany); G. Schriever, XTREME technologies GmbH (Germany); M. Corthout, Philips Extreme UV GmbH (Germany)

\section{EMERGENT IMAGING TECHNOLOGY}

714011 A novel curve-fitting procedure for determining proximity effect parameters in electron beam lithography [7140-53]

C.-H. Liu, H.-T. Ng, P. C. W. Ng, K.-Y. Tsai, National Taiwan Univ. (Taiwan); S.-J. Lin, J.-H. Chen, Taiwan Semiconductor Manufacturing Co., Ltd. (Taiwan)

$71401 \mathrm{~J} \mathrm{Calculation} \mathrm{of} \mathrm{three-dimensional} \mathrm{profiles} \mathrm{of} \mathrm{photoresist} \mathrm{exposed} \mathrm{by} \mathrm{localized} \mathrm{electric} \mathrm{fields}$ of high-transmission metal nano-apertures [7140-54]

E. Lee, J. W. Hahn, Yonsei Univ. (Korea, Republic of)

$71401 \mathrm{~K}$ Study on imaging characterization of ArF high index immersion lithography [7140-55]

S. Park, J.-T. Park, K. Lee, T.-S. Eom, J.-S. Kim, H.-S. Kim, S.-C. Moon, Hynix Semiconductor Inc. (Korea, Republic of)

\section{EUV INFRASTRUCTURE}

7140 iN Influences of various defects on extreme ultraviolet mask [7140-58]

E.-J. Kim, J.-H. You, Hanyang Univ. (Korea, Republic of); J.-Y. Lee, D.-B. Kim, J.-H. Kim, Dongjin Semichem Co., Ltd. (Korea, Republic of); H.-K. Oh, Hanyang Univ. (Korea, Republic of)

$71401 Q \quad$ Cost of ownership for future lithography technologies [7140-61]

A. J. Hazelton, Nikon Corp. (Japan); A. Wüest, G. Hughes, L. C. Litt, F. Goodwin, SEMATECH (United States)

\section{LITHO MASK TECHNOLOGY}

7140 is Removal of particles from lithographic masks through plasma-assisted cleaning by metastable atomic neutralization [7140-63]

W. M. Lytle, D. S. Szybilski, C. E. Das, R. Raju, V. Surla, M. J. Neumann, D. N. Ruzic, Univ. of Illinois at Urbana-Champaign (United States)

7140 IT Application of multi-tone mask technology in photolithographic fabrication of color filter components in LCD [7140-65]

Y. Takada, M. Fukui, T. Sai, Mitsubishi Chemical Corp. (Japan)

$71401 \mathrm{~V}$ Effective solution to reticle haze formation at 193nm lithography [7140-67] W.-J. Tseng, S.-H. Chiou, Rexchip Electronics Corp. (Taiwan); M.-C. Chiu, P.-S. Lee, Gudeng Precision Industrial Co., Ltd. (Taiwan)

7140 IW Robust mask design with defocus variation using inverse synthesis (Best Student Paper Award) [7140-88]

N. Jia, The Univ. of Hong Kong (Hong Kong China); A. K. Wong, Magma Design Automation (United States); E. Y. Lam, The Univ. of Hong Kong (Hong Kong China) 
$71401 \mathrm{X}$ A methodology for double patterning compliant split and design (Invited Paper) [7140-68] V. Wiaux, S. Verhaegen, IMEC (Belgium); F. Iwamoto, Panasonic (Japan); M. Maenhoudt, IMEC (Belgium); T. Matsuda, Panasonic (Japan); S. Postnikov, Infineon (Germany); G. Vandenberghe, IMEC (Belgium)

714012 Full-chip pitch/pattern splitting for lithography and spacer double patterning technologies [7140-70] T.-B. Chiou, ASML Taiwan Ltd. (Taiwan); R. Socha, ASML (United States); H.-Y. Kang, ASML Korea Co., Ltd. (Korea, Republic of); A. C. Chen, ASML Taiwan Ltd. (Taiwan); S. Hsu, H. Chen, L. Chen, ASML Brion (United States)

714020 Alternative technology for double patterning process simplification [7140-71] H.-Y. Lim, K.-Y. Jang, J.-H. Kim, S.-G. Lee, S. Park, T.-H. Kim, C.-K. Bok, S.-C. Moon, Hynix Semiconductor Inc. (Korea, Republic of)

714021 Challenges of $29 \mathrm{~nm}$ half-pitch NAND Flash STI patterning with 193nm dry lithography and self-aligned double patterning [7140-72]

M. C. Chiv, B. S.-M. Lin, M. F. Tsai, Y. S. Chang, M. H. Yeh, T. H. Ying, Powerchip Semiconductor Corp. (Taiwan); C. Ngai, J. Jin, S. Yuen, S. Huang, Y. Chen, L. Miao, K. Tai, A. Conley, I. Liu, Applied Materials, Inc. (United States)

\section{OPTICAL (DPT PROCESS)}

714022 Mask specification guidelines in spacer patterning technology [7140-73]

K. Hashimoto, H. Mukai, S. Miyoshi, S. Yamaguchi, H. Mashita, Y. Kobayashi, K. Kawano,

T. Hirano, Toshiba Corp. (Japan)

714023 Cluster optimization to improve CD control as an enabler for double patterning [7140-74]

L. Tedeschi, C. Rosslee, Sokuda Co., Ltd. (United States); D. Laidler, P. Leray, K. D'havé, IMEC (Belgium)

714024 CD uniformity improvement for $3 \times$ nm node devices [7140-75]

C.-M. Park, H.-B. Kim, H.-D. Kim, H.-W. Kim, C.-H. Kim, J.-O. Park, D. Goo, J. Yeo, S.-W. Choi, W.-S. Han, SAMSUNG Electronics Co., Ltd. (Korea, Republic of); M.-S. Choi, S.-W. Hwang, Korea Univ. (Korea, Republic of)

714025 Using scatterometry to improve process control during the spacer pitch splitting process [7140-76]

S. Corboy, C. MacNaughton, T. Gubiotti, M. Wollenweber, KLA-Tencor Corp. (United States)

\section{ADVANCED EXPOSURE TOOL CONTROL}

$714027 \quad$ Experimental proximity matching of ArF scanners [7140-78]

J. Bekaert, L. Van Look, P. De Bisschop, J. Van de Kerkhove, G. Vandenberghe, IMEC VZW (Belgium); K. Schreel, J. Menger, G. Schiffelers, E. Knols, H. van der Laan, R. Willekers, ASML (Netherlands) 
714028 Exposure tool for 32-nm lithography: requirements and development progress [7140-79] A. J. Hazelton, J. Ishikawa, N. Magome, Nikon Corp. (Japan)

$71402 \mathrm{~A}$ The impact of illuminator signatures on optical proximity effects [7140-81]

J. K. Tyminski, S. P. Renwick, Nikon Precision Inc. (United States)

OPTICAL (OPC)

7140 2B Model based pattern matching [7140-82]

L. S. Melvin III, J. Tuttle, M. Boman, Synopsys, Inc. (United States)

7140 2D Optimization of RET flow using test layout [7140-84]

Y. Zhang, S. Sethi, K. Lucas, Synopsys, Inc. (United States)

7140 2E Image parameter-based scatter bar optimization [7140-85]

R. Chou, L.-T. Hsiao, H. Y. Liao, J. Lin, R. Shen, J. Schacht, Mentor Graphics Taiwan, Ltd.

(Taiwan); D. Chou, S. Jayaram, P. LaCour, Mentor Graphics Corp. (United States)

$71402 \mathrm{G}$ The divergence of image and resist process metrics [7140-45]

J. J. Biafore, S. Kapasi, S. A. Robertson, M. D. Smith, KLA-Tencor, FINLE (United States)

\section{NOVEL RESIST MATERIAL AND PROCESSING}

$71402 \mathrm{H} \quad$ Pattern freezing process free litho-litho-etch double patterning (Invited Paper) [7140-90] T. Ando, M. Takeshita, R. Takasu, Y. Yoshii, J. Iwashita, S. Matsumaru, S. Abe, T. Iwai, Tokyo Ohka Kogyo Co., Ltd. (Japan)

$714021 \quad$ Novel embedded barrier layer materials for ArF non-topcoat immersion applications [7140-91]

D. Wang, C. WU, C. B. XU, G. Barclay, P. Trefonas, S. Dinglee, Rohm and Haas Electronic Materials (United States)

$71402 \mathrm{~J} \quad$ Reactive liquid crystal materials for optically anisotropic patterned retarders [7140-93] R. Harding, I. Gardiner, Merck (United Kingdom); H.-J. Yoon, Merck (United Kingdom) and Merck Advanced Technologies (Korea, Republic of); T. Perrett, O. Parri, K. Skjonnemand, Merck (United Kingdom)

7140 2K Development status of High OD photo resist for CF black matrix [7140-94]

D. Shiota, A. Katano, M. Shida, K. Uchikawa, Tokyo Ohka Kogyo Co., Ltd. (Japan)

\section{NOVEL RESIST MATERIAL AND PROCESSING II}

$71402 \mathrm{M}$ Exposure illuminance dependability in the lithography characteristic of color resist for LCD color filter [7140-96]

M. Yashiro, M. Ohta, Toyo Ink Mfg. Co., Ltd. (Japan)

714020 Development of photosensitive silsesquioxane [7140-98]

Y. Tashiro, T. Sekito, T. Iwata, D. Yokoyama, T. Nonaka, AZ Electronic Materials Japan (Japan) 
7140 2P Grazing incidence ion beams for reducing LER [7140-99]

C. R. M. Struck, M. J. Neumann, R. Raju, Univ. of Illinois at Urbana-Champaign (United States); R. L. Bristol, Intel Corp. (United States); D. N. Ruzic, Univ. of Illinois at Urbana-Champaign (United States)

$71402 Q \quad$ Use of spin-on-hard mask materials for nano scale patterning technology [7140-109] W.-H. Wu, E. Y. Chang, National Chiao Tung Univ. (Taiwan); H.-S. Cheon, S. K. Kim, H. M. Cho, K.-H. Yoon, J. S. Kim, T. Chang, S. Shin, Samsung Cheil Industries, Inc. (Korea, Republic of)

\section{ADVANCED MATERIALS}

7140 2R Development of spin-on carbon hardmasks with comparable etch resistance to Amorphous Carbon Layer (ACL) [7140-100] H.-S. Cheon, K.-H. Yoon, M.-S. Kim, S. B. Oh, J. Y. Song, N. Tokareva, J. S. Kim, T. Chang, SAMSUNG Cheil Industries, Inc. (Korea, Republic of)

$71402 S \quad H i g h$ Si content BARC for applications in dual BARC systems such as tri-layer patterning [7140-101]

J. Kennedy, S.-Y. Xie, Z.-Y. Wu, R. Katsanes, K. Flanigan, Honeywell Co., Ltd. (United States);

K. Lee, Honeywell Co., Ltd. (Taiwan); M. Slezak, N. Fender, JSR Micro, Inc. (United States);

J. Takahashi, JSR Inc. (Taiwan)

$71402 \mathrm{~T} \quad$ Spin-on trilayer scheme: enabling materials for extension of ArF immersion lithography to 32nm node and beyond [7140-102]

R. Zhang, A. G. Timko, L. Pylneva, J. Loch, H. Wu, D. J. Abdallah, R. A. Collett, Y. Wei,

D. Rahman, D. S. McKenzie, P.-H. Lu, M. Neisser, AZ Electronic Materials (United States)

$71402 U$ BARC technology for 1.35 NA lithography [7140-103]

M. Reilly, G. G. Zhang, K. Spizuoco, Rohm and Haas Electronic Materials (United States)

$71402 \mathrm{~V}$ Optimization of optical properties of silicon-based anti-reflective spin-on hardmask materials [7140-104]

S. K. Kim, H. M. Cho, C. Woo, S. R. Koh, M. Kim, H. C. Yoon, W. Lee, S.-W. Shin, J. S. Kim,

T. Chang, SAMSUNG Cheil Industries, Inc. (Korea, Republic of)

\section{ADVANCED MATERIALS II}

$71402 \mathrm{~W} \quad$ Acid-degradable hyperbranched polymer and its application in bottom anti-reflective coatings [7140-106]

R.-M. L. Mercado, H. Xu, J. A. Lowes, J. D. Meador, D. J. Guerrero, Brewer Science, Inc. (United States)

$71402 \mathrm{X} \quad$ Bottom anti-reflective coating for hyper NA process: theory, application, and material development [7140-108]

H. Yao, J. Cho, J. Yin, S. Mullen, G. Lin, M. Neisser, R. Dammel, AZ Electronic Materials US Corp. (United States)

$71402 Y \quad$ A technique for rapid elimination of microbubbles for photochemical filter startup [7140-110] A. Wu, W. Chow, Entegris, Inc. (United States) 
714022 The first on-site evaluation of a new filter optimized for TARC and developer [7140-111] T. Umeda, Nihon Pall, Ltd. (Japan); T. Ishibashi, Renesas Technology Corp. (Japan); A. Nakamura, J. Ide, M. Nagano, K. Omura, Renesas Semiconductor Engineering Corp. (Japan); S. Tsuzuki, T. Numaguchi, Nihon Pall, Ltd. (Japan)

\section{POSTER SESSION}

714030 Optimization of mask manufacturing rule check constraint for model based assist feature generation [7140-64]

S. Shim, Y. Kim, Y. Chun, S. Lee, S. Lee, S. Choi, W. Han, S. Chang, S. Yoon, H. Kim, W. Ki,

S. Woo, H. Cho, SAMSUNG Electronics Co., Ltd. (Korea, Republic of)

714031 The noble resists composed of cationic and anionic polymerizable PAGs [7140-92]

J. H. Oh, D. C. Seo, H. S. Joo, S. D. Jung, J. H. Kim, S. J. Lee, R. R. Park, J. Han, J. H. Park, Korea Kumho Petrochemical Co., Ltd. (Korea, Republic of)

714032 Practical requirement for reflectivity control in sub 30nm device using high NA immersion lithography [7140-105]

Y.-K. Jang, S. Han, H. Kim, J.-Y. Yoon, S. Lee, K. Yoon, S. Oh, S.-W. Choi, W.-S. Han, SAMSUNG Electronics Co., Ltd. (Korea, Republic of)

714033 Application of exposure simulation system to reduce isolated-dense bias by using annular off-axis illumination [7140-112]

Y.-N. Shih, Yuan Ze Univ. (Taiwan) and Photronics Semiconductor Mask Corp. (Taiwan);

N.-P. Chen, Yuan Ze Univ. (Taiwan)

714034 Resolution enhancement techniques for contact hole printing of sub-50nm memory device [7140-113]

H. Shin, T. You, M. Yoo, J. Choi, K. Yang, C. Park, D. Yim, Hynix Semiconductor Inc. (Korea,

Republic of)

714035 Process capability comparison between LELE DPT and spacer for NAND flash 32nm and below [7140-114]

S. Tseng, A. C. Chen, ASML (Taiwan)

714037 A cost effective spin on sidewall material alternative to the CVD sidewall process [7140-116] D. Maruyama, B.-C. Ho, S. Yoon, R. Sakamoto, Y. Sakaida, K. Hashimoto, N. Fujitani, H. Yaguchi, K. Matsubara, Nissan Chemical Industries, Ltd. (Japan)

$714038 \quad 22 \mathrm{~nm} \mathrm{1:1} \mathrm{line} \mathrm{and} \mathrm{space} \mathrm{patterning} \mathrm{by} \mathrm{using} \mathrm{double} \mathrm{patterning} \mathrm{and} \mathrm{resist} \mathrm{reflow} \mathrm{process}$ [7140-117]

J.-M. Park, J.-H. Yoo, J.-Y. Hong, I. An, H.-K. Oh, Hanyang Univ. (Korea, Republic of)

714039 193-nm immersion lithography for high volume manufacturing using novel immersion exposure tool and coater/developer system [7140-118]

S. Wakamizu, H. Kyouda, Tokyo Electron Kyushu Ltd. (Japan); K. Nakano, T. Fujiwara, Nikon Corp. (Japan) 
7140 3A Defect transfer from immersion exposure process to etching process using novel immersion exposure and track system [7140-119]

O. Miyahara, H. Kosugi, Tokyo Electron Kyushu Ltd. (Japan); S. Dunn, Tokyo Electron Ltd. Technology Ctr., America, LLC (United States); Y. van Dommelen, C. Grouwstra, ASML

Netherlands B.V. (Netherlands)

$71403 C$ The limit to 4X EUVL [7140-121]

K. Takehisa, Lasertec Corp. (Japan)

7140 3D Correction for surface charge induced beam displacement in large area sub- $45 \mathrm{~nm}$ patterning with FIB lithography [7140-122]

M. Chung, Southern Taiwan Univ. (Taiwan) and National Cheng Kung Univ. (Taiwan);

H.-Y. Lin, J.-H. Tsai, Industrial Technology Research Institute (Taiwan)

$71403 E \quad C D$ uniformity improvement of sub 60nm contact hole using model based OPC [7140-123]

H.-S. Yune, Y.-B. Ahn, J.-C. Kim, H.-J. Shin, G. Yoo, J. Moon, B.-S. Nam, D. Yim, Hynix

Semiconductor Inc. (Korea, Republic of)

$71403 \mathrm{~F}$ Analysis of adsorption effect of absorbent and threshold limit value (TLV) of Q-time for 193nm ArF reticle haze resistance [7140-124]

F.-S. Chu, S.-H. Chiou, Rexchip Electronics Corp. (Taiwan)

$71403 G$ The APC (Advanced Process Control) procedure for process window and CDU improvement using DBMs [7140-125]

J. Kim, T. Lee, A. Jung, G. Yoo, H. Yang, D. Yim, S. Park, Hynix Semiconductor Inc. (Korea, Republic of); J. Seo, B. Park, T. Hasebe, M. Yamamoto, NanoGeometry Research Inc. (Japan)

$71403 \mathrm{H} \quad$ Application automatic focusing with DVD pickup head in FPD substrate inspection [7140-126]

C. M. Tseng, National Sun Yat-Sen Univ. (Taiwan) and Metal Industries Research and Development Ctr. (Taiwan); C. Y. Chung, Metal Industries Research and Development Ctr. (Taiwan); S. C. Chiu, C. W. Yen, National Sun Yat-Sen Univ. (Taiwan)

7140 3J Developing loading effect on lithography I-line process [7140-128] T. Huang, W. Wang, C.-Y. Huang, N. Tseng, T.-J. Guo, C.-L. Shih, W.-B. Wu, Nanya Technologies Corp. (Taiwan)

7140 3K Novel process proximity correction by the pattern-to-pattern matching method with DBM [7140-129]

D. Park, J. Choi, H. Yune, J. Choi, C. Kim, Hynix Semiconductor Inc. (Korea, Republic of); B.-R. Choi, ASML Korea Co., Ltd (Korea, Republic of); D. Yim, Hynix Semiconductor Inc. (Korea, Republic of)

$71403 \mathrm{~N}$ Lithography performance and simulation accuracy at different polarization states for sub 40nm node [7140-132]

K.-Y. Park, ASML Korea Co., Ltd. (Korea, Republic of); H.-Y. Kang, ASML (United States); G. Isai, K. Elbattay, P. van Oorschot, S. Young, ASML Netherlands B.V. (Netherlands); S.-C. Oh, Y.-H. Min, S.-G. Hong, Y.-T. Park, ASML Korea Co., Ltd. (Korea, Republic of) 
$71403 R \quad$ Considering mask pellicle effect for more accurate OPC model at 45nm technology node [7140-136]

C.-H. Wang, Q. Liu, Semiconductor Manufacturing International Corp. (China); L. Zhang, Mentor Graphics Corp. (China)

$71403 \mathrm{U}$ Acid diffusion length dependency for 32-nm node attenuated and chromeless phase shift mask [7140-139]

J.-H. You, Y.-M. Kang, M. Jung, H.-K. Oh, Hanyang Univ. (Korea, Republic of)

$71403 X \quad$ Cycloolefin effect in cycloolefin-(meth)acryl copolymer [7140-142]

H. S. Lim, D. C. Seo, C. S. Lee, S. W. Park, S. J. Kim, D. H. Shin, J. B. Shin, J. H. Park, Korea Kumho Petrochemical Co., Ltd. (Korea, Republic of)

$71403 Y \quad$ Spacer double patterning technique for sub-40nm DRAM manufacturing process development [7140-143]

W. Shiu, W. Ma, H. W. Lee, J. S. Wu, Y. M. Tseng, K. Tsai, C. T. Liao, A. Wang, A. Yau, Y. R. Lin, Y. L. Chen, T. Wang, W. B. Wu, C. L. Shih, Nanya Technology Corp. (Taiwan)

$71403 Z$ Development of multi-layer process materials for hyper-NA lithography process [7140-144] Y. Sakaida, M. Nakajima, T. Shinjo, K. Hashimoto, Nissan Chemical Industries, Ltd. (Japan)

$714041 \quad$ CD control enhancement by laser bandwidth stabilization for advanced lithography application [7140-146]

R. C. Peng, T. Wu, K. W. Chang, C. P. Yeh, H. H. Liu, H. J. Lee, J. Lin, Taiwan Semiconductor Manufacturing Corp. (Taiwan); A. Chang, B. S.-M. Lin, Cymer Inc. (Taiwan)

714042 Analysis of the effect of laser bandwidth on imaging of memory patterns [7140-147] N. Seong, Cymer, Inc. (United States); I. Kim, D. Kang, S.-H. Lee, J. Choi, SAMSUNG Electronics Co., Ltd. (Korea, Republic of)

714043 Throughput improvement from routing reduction by using CPE (correction per exposure) [7140-148]

R. C. Chang, J.-C. Yang, C.-H. Chen, C.-C. Lin, Inotera Memories Inc. (Taiwan); C. Wang, W. Lin, C.-C. Chen, ASML Taiwan Ltd. (Taiwan)

Author Index 
Downloaded From: https://www.spiedigitallibrary.org/conference-proceedings-of-spie on 26 Apr 2023

Terms of Use: https://www.spiedigitallibrary.org/terms-of-use 


\title{
Conference Committee
}

\author{
Conference Chair
}

Alek C. Chen, ASML Taiwan Ltd. (Taiwan)

Conference Cochairs

Burn Lin, Taiwan Semiconductor Manufacturing Co., Ltd. (Taiwan)

Anthony Yen, Taiwan Semiconductor Manufacturing Co., Ltd. (Taiwan)

Program Committee Chair

Woo Sung Han, SAMSUNG Electronics Co., Ltd. (Korea, Republic of)

Program Committee Cochair

Geert Vandenberghe, IMEC (Belgium)

Program Committee

Nigel R. Farrar, Cymer, Inc. (United States)

Donis G. Flagello, ASML, Inc. (United States)

Kohji Hashimoto, Toshiba Corp. (Japan)

Naoya Hayashi, Dai Nippon Printing Co., Ltd. (Japan)

Tokuyuki Honda, Canon Inc. (Japan)

Peter Huang, United Microelectronics Corp. (Taiwan)

Hideki Ina, Canon Inc. (Japan)

Soichi Inove, Toshiba Corp. (Japan)

Masaomi Kameyama, Nikon Corp. (Japan)

Hoyoung Kang, ASML Korea Co., Ltd. (United States)

Nelson Lai, Powerchip Semiconductor Corp. (Taiwan)

Edmund Yin-Mum Lam, The University of Hong Kong (Hong Kong China)

Benjamin Szu-Min Lin, Powerchip Semiconductor Corp. (Taiwan)

Hyekeun Oh, Hanyang University (Korea, Republic of)

Junichi Onodera, Tokyo Ohka Kogyo Co., Ltd. (Japan)

Christopher J. Progler, Photronics, Inc. (United States)

Kuen-Yu Tsai, National Taiwan University (Taiwan)

Wen-Bin Wu, AU Optronics Corp. (Taiwan)

Dong-Gyu Yim, Hynix Semiconductor Inc. (Korea, Republic of)

Gary Zhang, Rohm and Haas Electronic Materials (United States)

Session Chairs

1 Plenary Session

Alek C. Chen, ASML Taiwan Ltd. (Taiwan) 
EUVL Technology

Kuen-Yu Tsai, National Taiwan University (Taiwan)

Kohji Hashimoto, Toshiba Corp. (Japan)

3 Emergent Lithographic Technology

Masaomi Kameyama, Nikon Corp. (Japan)

Hye-Keun Oh, Hanyang University (Korea, Republic of)

4 CD Metrology

Hideki Ina, Canon Inc. (Japan)

Vincent Wiaux, IMEC (Belgium)

5 Process Control and Metrology

Kafai Lai, IBM Microelectronics Div. (United States)

Tsann-Bim Chiou, ASML Taiwan Ltd. (Taiwan)

$6 \quad$ Lithography Process Control

Michael E. Adel, KLA-Tencor Israel (Israel)

Masaomi Kameyama, Nikon Corp. (Japan)

7 Defect Inspection

Edmund Y. Lam, The University of Hong Kong (Hong Kong, China)

Jan C. H. Mulkens, ASML Netherlands B.V. (Netherlands)

8 Optical (Imaging)

Hideki Ina, Canon Inc. (Japan)

Vincent Wiaux, IMEC (Belgium)

9 Optical (Imaging) II

Kohji Hashimoto, Toshiba Corp. (Japan)

Tsann-Bim Chiou, ASML Taiwan Ltd. (Taiwan)

10 EUV Scanner and Sources

Jack J. H. Chen, Taiwan Semiconductor Manufacturing Co. Ltd. (Taiwan)

Kuen-Yu Tsai, National Taiwan University (Taiwan)

11 Emergent Imaging Technology

Nigel R. Farrar, Cymer, Inc. (United States)

Hye-Keun Oh, Hanyang University (Korea, Republic of)

12 EUV Infrastructure

Eric Hendrickx, IMEC (Belgium)

Jack J. H. Chen, Taiwan Semiconductor Manufacturing Co. Ltd. (Taiwan) 
13 Litho Mask Technology

Naoya Hayashi, Dai Nippon Printing Co., Lłd. (Japan)

Hoyoung Kang, ASML Korea Co., Ltd. (Korea, Republic of)

14 Optical (DPT)

Joost P. M. Bekaert, IMEC (Belgium)

Kuen-Yu Tsai, National Taiwan University (Taiwan)

15 Optical (DPT Process)

Hideki Ina, Canon Inc. (Japan)

Ho-Young Kang, ASML Korea Co., Ltd. (Korea, Republic of)

16 Advanced Exposure Tool Control

Jan C. H. Mulkens, ASML Netherlands B.V. (Netherlands)

Nigel R. Farrar, Cymer, Inc. (United States)

17 Optical (OPC)

Tsann-Bim Chiou, ASML Taiwan Ltd. (Taiwan)

Eric Hendrickx, IMEC (Belgium)

18 Novel Resist Material and Processing

Junichi Onodera, Tokyo Ohka Kogyo Co., Ltd. (Japan)

Wen-Yi Lin, AU Optronics Corp. (Taiwan)

19 Novel Resist Material and Processing II

Charles Chang, Taiwan Semiconductor Manufacturing Co. Ltd.

(Taiwan)

Shuji S. Dinglee, Rohm and Haas Electronic (Taiwan)

20 Advanced Materials

Charles Chang, Taiwan Semiconductor Manufacturing Co. Ltd.

(Taiwan)

Wen-Yi Lin, AU Optronics Corp. (Taiwan)

21 Advanced Materials II

Junichi Onodera, Tokyo Ohka Kogyo Co., Ltd. (Japan)

Shuji S. Dinglee, Rohm and Haas Electronic (Taiwan) 
Downloaded From: https://www.spiedigitallibrary.org/conference-proceedings-of-spie on 26 Apr 2023

Terms of Use: https://www.spiedigitallibrary.org/terms-of-use 